\title{
Linear Stability Analysis of Runge-Kutta Methods for Singular Lane-Emden Equations
}

\author{
M. O. Ogunniran ${ }^{\mathrm{a}, *}$, O. A. Tayo ${ }^{\mathrm{a}}$, Y. Haruna ${ }^{\mathrm{b}}$, A. F. Adebisi ${ }^{\mathrm{a}}$ \\ ${ }^{a}$ Department of Mathematical Sciences, Osun State University, Osogbo \\ ${ }^{b}$ Department of Mathematics, Saadatu Rimi College of Education Kumbotso, Kano State
}

\begin{abstract}
Runge-Kutta methods are efficient methods of computations in differential equations, the classical Runge-Kutta method of order 4 happens to be the most popular of these methods, and most times it is attached to the mind when Runge-Kutta methods are mentioned. However, there are numerous forms of them existing in lower and higher orders of the classical method. This work investigates the linear stabilities and abilities of some selected explicit members of these Runge-Kutta methods in integrating the singular Lane-Emden differential equations. The results obtained established the ability of the classical Runge-Kutta method and why is mostly used in computations.
\end{abstract}

Keywords: Classical, Stability, Explicit, Lane-Emden equations, Ordinary Differential Equations

Article History :

Received: 13 April 2020

Received in revised form: 30 May 2020

Accepted for publication: 01 June 2020

Published: 01 August 2020

(C)2020 Journal of the Nigerian Society of Physical Sciences. All rights reserved. Communicated by: T. Latunde

\section{Introduction}

The most popular of the methods of Runge-Kutta is the classical Runge-Kutta method of order 4, 'classical' as related to the method obtained in the pre-computer era. However, many other forms of Runge-Kutta methods have been derived by numerous authors in the past. Existing methods of Runge-Kutta are of orders 2, 3, and orders greater than 4. Methods of orders greater than 4 are regarded as higher-order methods of RungeKutta. The numerical solutions of singular Initial Value Problems (IVP) and Boundary Value Problems (BVP) of secondorder ordinary differential equations (ODEs) have been studied in this work. Existing methods for the singular problems are

*Corresponding author tel. no: +2347039104606

Email address: muideen. ogunniran@uniosun.edu.ng (M. O. Ogunniran ) series, analytical methods and of recent non-series numerical methods as obtained by various authors.

The general form of second-order singular differential equations is denoted as:

$$
y^{\prime \prime}(x)+\frac{P(x)}{Q(x)} y^{\prime}(x)+f(x, y)=g(x, y) ; \quad a \leq x \leq b .
$$

To solve equation (1), any of the conditions stated below need to be imposed.

$$
\begin{aligned}
& y(a)=\alpha, y^{\prime}(a)=\beta \\
& y(a)=\alpha_{1}, y^{\prime}(b)=\beta_{1}
\end{aligned}
$$

Equation (1) together with (2) are called Initial Value Problems (IVPs) while equations (1) and (3) are called Boundary Value Problems (BVPs).

Equation (1) is singular at $Q(x)=0$, and $f(x, y), g(x, y)$ are nonlinear continuous functions. It is well known that some of these 
problems have proved to be either difficult to solve or cannot be solved analytically due to the singularity as the approximate solutions lost their accuracy in the neighbourhood of the singular points. This discontinuous property has raised more interest in many applied Mathematicians and Physicists in the studies of this problem. Huta [1] developed the 8-stage Runge-Kutta method of Order 6 in solving differential equation problems, $\mathrm{Qu}$ and Agwarwal [2] employed collocation method for solving a class of singular non-linear two-point BVP. Koch, Peter \& Ewa [3] evaluated the approximate solution of the singular IVP by implicit Euler method and finally used an acceleration technique known as the iterated defect correction to improve the approximations. Wazwaz $[4,5]$ presented series and exact solution of Lane-Emden and Emden-Fowler type of problems based on Adomian decomposition and modified Adomian decomposition methods. A Simplified Derivation and Analysis of Fourth Order Runge Kutta Method was presented by Musa, Ibrahim, \& Waziri [6]. Roul [7] presented a new efficient recursive technique for solving singular boundary value problems arising in various physical models. Abbas Al-Shimmary [8] used the Runge-Kutta Method of order 6 to solve the initial value problem of ordinary differential equations. Roul [9] also presented a fourth-order B-spline collocation method and its error analysis for Bratu-type and Lane-Emden problems. A fastconverging iterative scheme for solving a system of Lane-Emden equations arising in catalytic diffusion reactions was presented by Madduri and Roul [10]. Roul [11] presented a fast converging iterative approach for the solution of doubly singular BVP with derivative dependent source foundation. Ogunniran, Haruna and Adeniyi [12] developed an efficient $k$-derivative method for Lane-Emden equations and related stiff problems, the paper focuses on the exploration of the possibility of a class of multi-derivative methods for approximating its solution. A new basic rational approximation method was developed for solving singular initial value problems of ordinary differential equations by Ogunniran \& Adeniyi [13]. Ogunniran [14] also developed a class of block multi-derivative numerical integrator for singular advection equations. The solution of EmdenFowler equations was solved using the variational iteration method by Olayiwola [15]. Olayiwola and Adegoke [16] presented the approach of homotopy perturbation with Laplace transform method in solving singular initial value problems. An optimal sixth-order quartic B-spline collocation method was developed by Roul, Thula and Goura [17] for solving Bratu-type and Lane-Emden-type problems. Roul, Madduri and Agarwal [18] developed a fast-converging recursive approach for Lane-Emden type initial value problems arising in astrophysics. Singh, Garg, Kanwar \& Ramos [19] developed an Efficient Optimized Adaptive step-size Hybrid Block Method for Integrating Differential System. This study aims at the exploration of the possibility of the classes of explicit Runge-Kutta methods in the solution of singular Lane-Emden problems of ordinary differential equations.

\section{Method}

The general m-stage Runge-kutta method is defined by

$$
\begin{aligned}
& y_{r+1}-y_{r}=h \theta\left(x_{r}, y_{r}, h\right), \\
& \theta(x, y, h)=\sum_{i=1}^{m} q_{i} \mu_{i} \\
& \mu_{1}=f(x, y) \\
& \mu_{r}=f\left(x+h p_{i}, y+h \sum_{s=1}^{i-1} b_{i s} \mu_{s}\right) ; i=2,3, \cdots, m \\
& p_{i}=\sum_{s=1}^{i-1} b_{i s}, i=2,3, \cdots, m ; h=x_{i}-x_{i-1}
\end{aligned}
$$

It can be observed that an m-stage Runge-kutta method involves $\mathrm{m}$-function evaluations per step. Each of the functions $\mu_{i}(x, y, h)$, $i=1,2,3, \cdots, m$ may be interpreted as an approximation of the derivative $y^{i}(x)$ and the function $\theta(x, y, h)$ as a weighted mean of these approximations.

There is a great deal of tedious manipulation involved in deriving Runge-Kutta methods of the higher order. However, the forms of Runge-Kutta methods in the scope of this work shall be given in the following section.

(i) 2-stage Runge-kutta method of Order 2 (RK2)

$$
\begin{gathered}
y_{i+1}-y_{i}=h \mu_{2} \\
\mu_{1}=f\left(x_{i}, y_{i}\right) \\
\mu_{2}=f\left(x_{i}+\frac{1}{2} h, y_{i}+\frac{1}{2} h \mu_{1}\right)
\end{gathered}
$$

(ii) 3-stage Runge-kutta method of Order 3 (RK3)

$$
\begin{gathered}
y_{i+1}-y_{i}=\frac{1}{6} h\left(\mu_{1}+4 \mu_{2}+\mu_{3}\right) \\
\mu_{1}=f\left(x_{i}, y_{i}\right) \\
\mu_{2}=f\left(x_{i}+\frac{1}{2} h, y_{i}+\frac{1}{2} h \mu_{1}\right) \\
\mu_{3}=f\left(x_{i}+h, y_{i}-h\left(\mu_{1}-2 \mu_{2}\right)\right)
\end{gathered}
$$

(iii) 4-stage Runge-kutta method of Order 4 (RK4)

$$
\begin{gathered}
y_{i+1}-y_{i}=\frac{1}{6} h\left(\mu_{1}+2 \mu_{2}+2 \mu_{3}+\mu_{4}\right) \\
\mu_{1}=f\left(x_{i}, y_{i}\right) \\
\mu_{2}=f\left(x_{i}+\frac{1}{2} h, y_{i}+\frac{1}{2} h \mu_{1}\right) \\
\mu_{3}=f\left(x_{i}+\frac{1}{2} h, y_{i}+\frac{1}{2} h \mu_{2}\right) \\
\mu_{4}=f\left(x_{i}+h, y_{i}+h \mu_{3}\right)
\end{gathered}
$$


(iv) 6-stage kutta-Nyström method of Order 5 (RK Nyström)

$$
\begin{gathered}
y_{i+1}-y_{i}=\frac{1}{192} h\left(23 \mu_{1}+125 \mu_{3}-81 \mu_{5}+125 \mu_{6}\right) \\
\mu_{1}=f\left(x_{i}, y_{i}\right) \\
\mu_{2}=f\left(x_{i}+\frac{1}{3} h, y_{i}+\frac{1}{2} h \mu_{1}\right) \\
\mu_{3}=f\left(x_{i}+\frac{2}{5} h, y_{i}+\frac{1}{25} h\left(4 \mu_{1}+6 \mu_{2}\right)\right) \\
\mu_{4}=f\left(x_{i}+h, y_{i}+\frac{1}{4} h\left(\mu_{1}-12 \mu_{2}+15 \mu_{3}\right)\right) \\
\mu_{5}=f\left(x_{i}+\frac{2}{3} h, y_{i}\right. \\
\left.+\frac{1}{81} h\left(6 \mu_{1}+90 \mu_{2}-50 \mu_{3}+8 \mu_{4}\right)\right) \\
\mu_{6}=f\left(x_{i}+\frac{4}{5} h, y_{i}\right. \\
\left.+\frac{1}{75} h\left(6 \mu_{1}+36 \mu_{2}+10 \mu_{3}+8 \mu_{4}\right)\right)
\end{gathered}
$$

(v) 8-stage Huta method of Order 6 (RK 8)

$$
\begin{gathered}
y_{i+1}-y_{i}=\frac{1}{840} h\left(4 \mu_{1}+216 \mu_{3}+27 \mu_{4}+272 \mu_{5}+27 \mu_{6}+216 \mu_{7}+41 \mu_{8}\right) \\
\mu_{1}=f\left(x_{i}, y_{i}\right) \\
\mu_{2}=f\left(x_{i}+\frac{1}{9} h, y_{i}+\frac{1}{9} h \mu_{1}\right) \\
\mu_{3}=f\left(x_{i}+\frac{1}{6} h, y_{i}+\frac{1}{24} h\left(\mu_{1}+3 \mu_{2}\right)\right) \\
\mu_{4}=f\left(x_{i}+\frac{1}{3} h, y_{i}+\frac{1}{6} h\left(\mu_{1}-3 \mu_{2}+4 \mu_{3}\right)\right) \\
\mu_{5}=f\left(x_{i}+\frac{1}{2} h, y_{i}+\frac{1}{8} h\left(-5 \mu_{1}+27 \mu_{2}-24 \mu_{3}+6 \mu_{4}\right)\right) \\
\mu_{6}=f\left(x_{i}+\frac{2}{3} h, y_{i}+\frac{1}{9} h\left(221 \mu_{1}-9813 \mu_{2}+867 \mu_{3}-102 \mu_{4}+\mu_{5}\right)\right) \\
\mu_{7}=f\left(x_{i}+\frac{5}{6} h, y_{i}+\frac{1}{48} h\left(-183 \mu_{1}+678 \mu_{2}-472 \mu_{3}-66 \mu_{4}+80 \mu_{5}+3 \mu_{6}\right)\right) \\
\mu_{8}=f\left(x_{i}+h, y_{i}+\frac{1}{82} h\left(716 \mu_{1}-2079 \mu_{2}+1002 \mu_{3}+834 \mu_{4}-454 \mu_{5}-9 \mu_{6}+72 \mu_{7}\right)\right)
\end{gathered}
$$

For purpose of completion and without loss of generality, special Runge-kutta methods which were developed to exhibit certain properties were also considered.

(vi) 5-stage Merson's Method of Order 4 (RK Merson)

$$
\begin{gathered}
y_{i+1}-y_{i}=\frac{1}{6} h\left(\mu_{1}+4 \mu_{4}+\mu_{5}\right) \\
\mu_{1}=f\left(x_{i}, y_{i}\right) \\
\mu_{2}=f\left(x_{i}+\frac{1}{3} h, y_{i}+\frac{1}{3} h \mu_{1}\right) \\
\mu_{3}=f\left(x_{i}+\frac{1}{3} h, y_{i}+\frac{1}{6} h\left(\mu_{1}+\mu_{2}\right)\right) \\
\mu_{4}=f\left(x_{i}+\frac{1}{2} h, y_{i}+\frac{1}{8} h\left(\mu_{1}+3 \mu_{3}\right)\right) \\
\mu_{5}=f\left(x_{i}+h, y_{i}+\frac{1}{2} h\left(\mu_{1}-3 \mu_{3}+4 \mu_{4}\right)\right)
\end{gathered}
$$

(vii) 5-stage Scraton's Method of Order 4 (RK Scraton)

$$
\begin{gathered}
y_{i+1}-y_{i}=h\left(\frac{17}{162} \mu_{1}+\frac{81}{170} \mu_{3}+\frac{32}{135} \mu_{4}+\frac{250}{1377} \mu_{5}\right) \\
\mu_{1}=f\left(x_{i}, y_{i}\right) \\
\mu_{2}=f\left(x_{i}+\frac{2}{9} h, y_{i}+\frac{2}{9} h \mu_{1}\right) \\
\mu_{3}=f\left(x_{i}+\frac{1}{3} h, y_{i}+\frac{1}{12} h\left(\mu_{1}+3 \mu_{2}\right)\right) \\
\mu_{4}=f\left(x_{i}+\frac{3}{4} h, y_{i}+\right. \\
\left.\frac{3}{128} h\left(23 \mu_{1}-81 \mu 2+90 \mu_{3}\right)\right) \\
\mu_{5}=f\left(x_{i}+\frac{9}{10} h, y_{i}+\right. \\
\left.\frac{9}{10000} h\left(-345 \mu_{1}+2025 \mu 2-1224 \mu_{3}+544 \mu_{4}\right)\right)
\end{gathered}
$$

(viii) 6-stage England's Method of Order 4 (RK England)

$$
\begin{gathered}
y_{i+1}-y_{i}=\frac{1}{6} h\left(\mu_{1}+4 \mu_{3}+\mu_{4}\right) \\
\mu_{1}=f\left(x_{i}, y_{i}\right) \\
\mu_{2}=f\left(x_{i}+\frac{1}{2} h, y_{i}+\frac{1}{2} h \mu_{1}\right) \\
\mu_{3}=f\left(x_{i}+\frac{1}{2} h, y_{i}+\frac{1}{4} h\left(\mu_{1}+\mu_{2}\right)\right) \\
\mu_{4}=f\left(x_{i}+h, y_{i}-h\left(\mu_{2}-2 \mu_{3}\right)\right) \\
\mu_{5}=f\left(x_{i}+\frac{2}{3} h, y_{i}+\right. \\
\left.\frac{1}{27} h\left(7 \mu_{1}+10 \mu_{2}+\mu_{4}\right)\right) \\
\mu_{6}=f\left(x_{i}+\frac{1}{5} h, y_{i}+\right. \\
\left.\frac{1}{625} h\left(28 \mu_{1}-125 \mu_{2}+546 \mu_{3}+54 \mu_{4}-378 \mu_{5}\right)\right)
\end{gathered}
$$

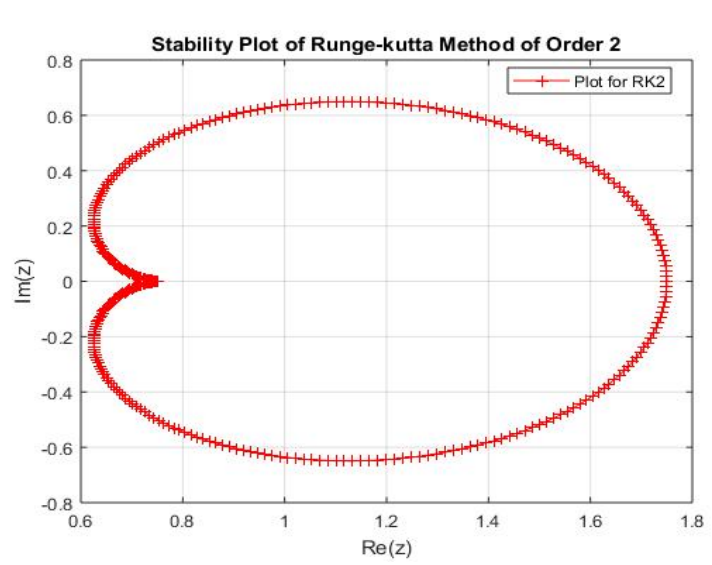

Figure 1. Stability Region for Runge-kutta Method of Order 2

\subsection{Linear Stability}

This is a behaviourial property related to $h>0$. As in most literature, the linear stability will be analyzed using the Dalquist's test

$$
y^{\prime}(t)=\gamma y(t), \quad \mathfrak{R}(\gamma)<0 .
$$

Applying methods (9)-(16) on (17), we have obtained a recurrence equation

$$
y_{i}=M(z) y_{i-1}
$$

where $M(z=h \gamma)$ for each of the method is given in Table 1 below The contour for regions of absolute stability as obtained from their characteristics equations are given in Figure 1 - 8 .

\section{Numerical Experiment}

This section contains the numerical example considered and their results presented in tables of absolute errors.

Example 1: Variable Coefficient Non-homogeneous Singular IVP [3]

$$
\left.\begin{array}{c}
y^{\prime \prime}(x)=-\frac{2}{x} y^{\prime}(x)-n^{2} \cos (n x) \\
-\frac{2}{x} n \sin (n x) ; y(0)=2, y^{\prime}(0)=0 \\
\text { Theoretical Solution : } y(x)=1+\cos (n x)
\end{array}\right\}
$$

* Absolute Error $=\mid$ Exact solution - Numerical solution $\mid, \mathrm{Nu}$ merical solution is obtained using the Runge-kutta methods.

Example 2: Variable Coefficient Homogeneous Singular Initial BVP [6]

$$
\begin{gathered}
\left(x^{2} y^{\prime}\right)^{\prime}=\beta\left(x y^{\prime}+y(\alpha+\beta-1)\right) x^{\alpha+\beta-2}, 0 \leq x \leq 1 \\
y(0)=1, y(1)=\exp (1) \\
\text { Theoretical Solution }=\exp \left(x^{4}\right)
\end{gathered}
$$


Table 1. Table of Characteristics Equations for Runge-kutta Methods

\begin{tabular}{|c|c|}
\hline Method & $M(z), z=h \lambda$ \\
\hline$(9)$ & $\frac{1}{4} z^{2}+\frac{1}{2} z+1$ \\
$(10)$ & $\frac{1}{6} z^{3}+\frac{1}{2} z^{2}+z+1$ \\
$(11)$ & $\frac{1}{24} z^{4}+\frac{1}{6} z^{3}+\frac{1}{2} z^{2}+z+1$ \\
$(12)$ & $\frac{1}{120} z^{5}+\frac{1}{24} z^{4}+\frac{1}{6} z^{3}+\frac{1}{2} z^{2}+z+1$ \\
$(13)$ & $\frac{z^{8}}{483840}+\frac{z^{7}}{4480}+\frac{z^{6}}{720}-\frac{179 z^{5}}{4032}-\frac{3049 z^{4}}{3360}-\frac{3119 z^{3}}{420}-\frac{3119 z^{2}}{140}+\frac{803 z}{840}+1$ \\
$(14)$ & $\frac{1}{36} z^{4}+\frac{1}{6} z^{3}+\frac{1}{2} z^{2}+z+1$ \\
$(15)$ & $\frac{1}{96} z^{5}+\frac{1}{24} z^{4}+\frac{1}{6} z^{3}+\frac{1}{2} z^{2}+z+1$ \\
$(16)$ & $\frac{1}{24} z^{4}+\frac{1}{6} z^{3}+\frac{1}{2} z^{2}+z+\frac{1}{12}$ \\
\hline
\end{tabular}

Table 2. Table of Absolute Errors for Example 1, $n=3$ at different $h$

\begin{tabular}{|c|c|c|c|c|c|c|c|c|c|}
\hline \multirow{2}{*}{$h$} & \multirow{2}{*}{$x$} & \multicolumn{8}{|c|}{$y(x)$} \\
\hline & & RK2 & RK3 & RK4 & RK Nyström & RK Merson & RK Scraton & RK England & RK8 \\
\hline \multirow{3}{*}{$10^{-1}$} & 0.2 & $9.1982 \times 10^{-2}$ & $9.0000 \times 10^{0}$ & $5.0392 \times 10^{-2}$ & $4.5167 \times 10^{-2}$ & $1.3355 \times 10^{-1}$ & $7.61289 \times 10^{0}$ & $5.0392 \times 10^{-2}$ & $1.1722 \times 10^{0}$ \\
\hline & 0.5 & $7.7896 \times 10^{-2}$ & $9.0000 \times 10^{0}$ & $2.0474 \times 10^{-2}$ & $7.5533 \times 10^{-2}$ & $1.6385 \times 10^{-1}$ & $8.3700 \times 10^{0}$ & $2.04738 \times 10^{-2}$ & $1.2308 \times 10^{0}$ \\
\hline & 0.7 & $8.1030 \times 10^{-2}$ & $9.0000 \times 10^{0}$ & $1.4680 \times 10^{-2}$ & $8.1382 \times 10^{-2}$ & $1.6970 \times 10^{-1}$ & $7.4117 \times 10^{0}$ & $1.4680 \times 10^{-2}$ & $1.2624 \times 10^{0}$ \\
\hline \multirow{3}{*}{$10^{-2}$} & 0.04 & $7.0148 \times 10^{-4}$ & $9.0000 \times 10^{0}$ & $2.5148 \times 10^{-4}$ & $7.0046 \times 10^{-4}$ & $1.5808 \times 10^{-3}$ & $9.7960 \times 10^{-1}$ & $2.5148 \times 10^{-4}$ & $1.2152 \times 10^{-2}$ \\
\hline & 0.4 & $5.7493 \times 10^{-4}$ & $9.0000 \times 10^{0}$ & $2.5188 \times 10^{-5}$ & $9.2689 \times 10^{-4}$ & $1.8072 \times 10^{-3}$ & $2.7924 \times 10^{0}$ & $2.5188 \times 10^{-5}$ & $3.9599 \times 10^{-2}$ \\
\hline & 0.9 & $7.5147 \times 10^{-4}$ & $9.0000 \times 10^{+1}$ & $1.1203 \times 10^{-5}$ & $9.4089 \times 10^{-4}$ & $1.8212 \times 10^{-3}$ & $1.200 \times 10^{-2}$ & $1.1203 \times 10^{-5}$ & $9.5724 \times 10^{-2}$ \\
\hline \multirow{3}{*}{$10^{-3}$} & 0.09 & $4.7178 \times 10^{-6}$ & $9.0000 \times 10^{0}$ & $1.1179 \times 10^{-7}$ & $9.4074 \times 10^{-6}$ & $1.8210 \times 10^{-5}$ & $8.2736 \times 10^{-1}$ & $1.1179 \times 10^{-7}$ & $1.6996 \times 10^{-3}$ \\
\hline & 0.19 & $4.8424 \times 10^{-6}$ & $9.0000 \times 10^{+1}$ & $5.2955 \times 10^{-8}$ & $9.4662 \times 10^{-6}$ & $1.8269 \times 10^{-5}$ & $1.5242 \times 10^{0}$ & $5.2955 \times 10^{-8}$ & $7.0497 \times 10^{-3}$ \\
\hline & 0.69 & $6.7846 \times 10^{-6}$ & $9.0000 \times 10^{+1}$ & $1.4584 \times 10^{-8}$ & $9.5046 \times 10^{-6}$ & $1.8307 \times 10^{-5}$ & $1.2185 \times 10^{0}$ & $1.4584 \times 10^{-8}$ & $6.5798 \times 10^{-2}$ \\
\hline Cmpt Time (s) & & 0.0025 & 0.0022 & 0.0035 & 0.0059 & 0.0039 & 0.0077 & 0.0065 & 0.021 \\
\hline
\end{tabular}

Table 3. Table of Absolute Errors for Example 2 Using $\alpha=2, \beta=4$

\begin{tabular}{|c|c|c|c|c|c|c|c|c|c|}
\hline \multirow[t]{2}{*}{$h$} & & \\
\hline & & RK2 & RK3 & RK4 & RK Nyström & RK Merson & RK Scraton & RK England & RK8 \\
\hline \multirow{3}{*}{$\frac{1}{10}$} & 0.2 & $1.5913 \times 10^{-3}$ & $1.5954 \times 10^{-3}$ & $1.5745 \times 10^{-3}$ & $1.5610 \times 10^{-3}$ & $1.5706 \times 10^{-3}$ & $7.0036 \times 10^{-4}$ & $1.5745 \times 10^{-3}$ & $1.5705 \times 10^{-3}$ \\
\hline & 0.5 & $5.8226 \times 10^{-2}$ & $5.8697 \times 10^{-2}$ & $5.7050 \times 10^{-2}$ & $5.7009 \times 10^{-2}$ & $5.7014 \times 10^{-2}$ & $2.5489 \times 10^{-2}$ & $5.7050 \times 10^{-2}$ & $5.7175 \times 10^{-2}$ \\
\hline & 0.8 & $3.8263 \times 10^{-1}$ & $3.8559 \times 10^{-1}$ & $3.7177 \times 10^{-1}$ & $3.7160 \times 10^{-1}$ & $3.7166 \times 10^{-1}$ & $5.7962 \times 10^{-1}$ & $3.7177 \times 10^{-1}$ & $3.7544 \times 10^{-1}$ \\
\hline \multirow{3}{*}{$\frac{1}{16}$} & 0.125 & $2.4357 \times 10^{-4}$ & $2.4382 \times 10^{-4}$ & $2.4258 \times 10^{-4}$ & $2.4231 \times 10^{-4}$ & $2.4234 \times 10^{-4}$ & $1.5826 \times 10^{-4}$ & $2.4258 \times 10^{-4}$ & $2.4233 \times 10^{-4}$ \\
\hline & 0.5 & $5.7534 \times 10^{-2}$ & $5.7757 \times 10^{-2}$ & $5.7026 \times 10^{-2}$ & $5.7019 \times 10^{-2}$ & $5.7020 \times 10^{-2}$ & $2.5563 \times 10^{-2}$ & $5.7026 \times 10^{-2}$ & $5.7237 \times 10^{-2}$ \\
\hline & 0.75 & $2.6863 \times 10^{-1}$ & $2.8731 \times 10^{-1}$ & $2.8304 \times 10^{-1}$ & $2.8302 \times 10^{-1}$ & $2.8303 \times 10^{-1}$ & $3.7761 \times 10^{-1}$ & $2.8304 \times 10^{-1}$ & $2.8600 \times 10^{-1}$ \\
\hline \multirow{3}{*}{$\frac{1}{32}$} & 0.25 & $3.8056 \times 10^{-3}$ & $3.8090 \times 10^{-3}$ & $3.7977 \times 10^{-3}$ & $3.7976 \times 10^{-3}$ & $3.7976 \times 10^{-3}$ & $1.1239 \times 10^{-3}$ & $3.7977 \times 10^{-3}$ & $3.8001 \times 10^{-3}$ \\
\hline & 0.5625 & $9.0304 \times 10^{-2}$ & $9.0405 \times 10^{-2}$ & $9.0077 \times 10^{-2}$ & $9.0076 \times 10^{-2}$ & $9.0077 \times 10^{-2}$ & $5.8501 \times 10^{-2}$ & $9.0077 \times 10^{-2}$ & $9.0639 \times 10^{-2}$ \\
\hline & 0.875 & $5.5592 \times 10^{-1}$ & $5.5618 \times 10^{-1}$ & $5.5376 \times 10^{-1}$ & $5.5538 \times 10^{-1}$ & $5.5376 \times 10^{-1}$ & $1.0939 \times 10^{0}$ & $5.5376 \times 10^{-1}$ & $5.6323 \times 10^{-1}$ \\
\hline Cmpt Time (s) & & 0.0023 & 0.0026 & 0.0042 & 0.0055 & 0.0033 & 0.0061 & 0.0055 & 0.011 \\
\hline
\end{tabular}




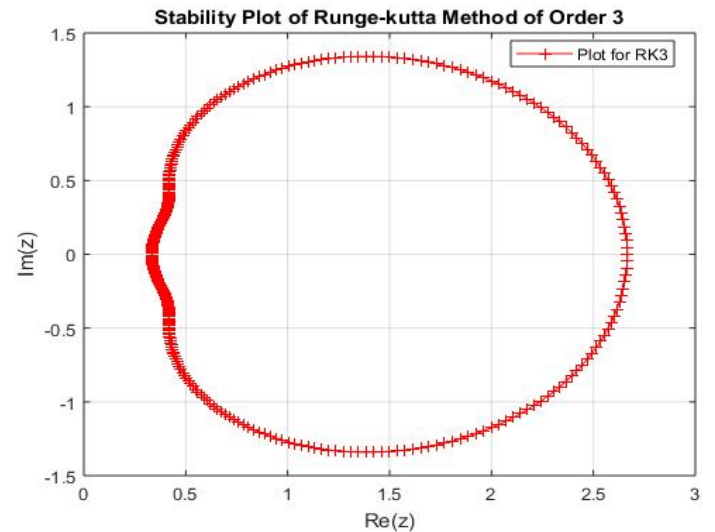

Figure 2. Stability Region for Runge-kutta Method of Order 3

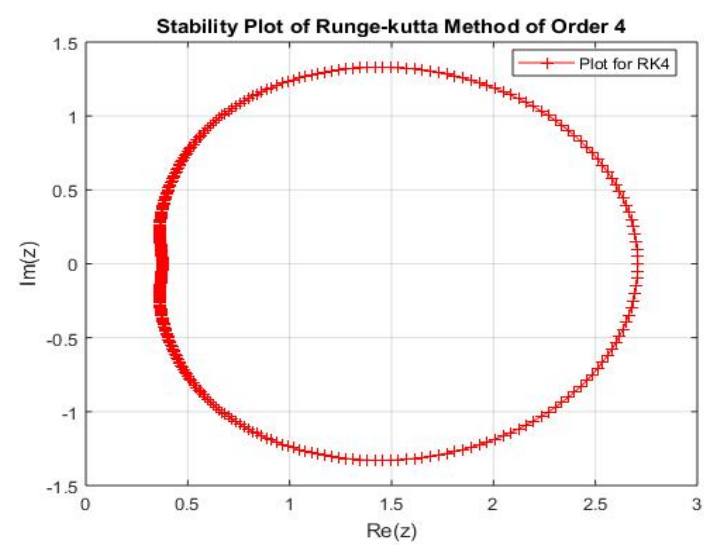

Figure 3. Stability Region for Runge-kutta Method of Order 4

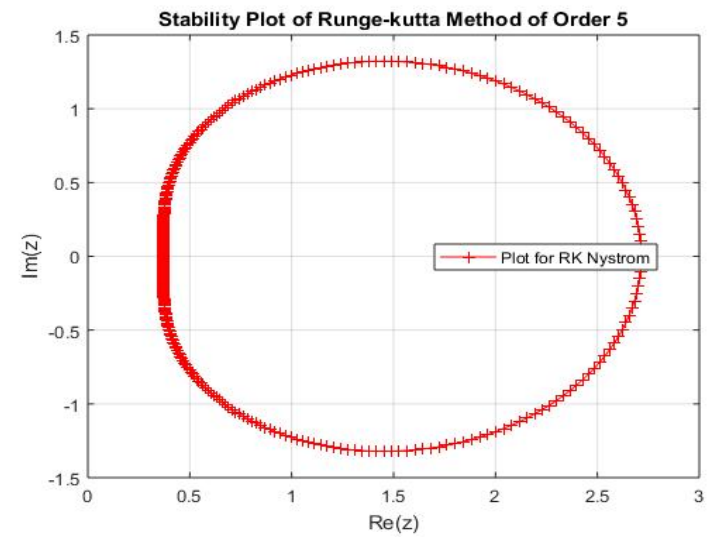

Figure 4. Stability Region for Kutta-Nyström Method

Example 3: Variable Coefficient Non-linear Homogeneous Singular IVP [3]

$$
\begin{gathered}
y^{\prime \prime}(x)=-\frac{2}{x} y^{\prime}(x)-y^{5}(x) ; x \in[0,1] y(0)=1, y^{\prime}(0)=0 \\
\text { Theoretical Solution : } y(x)=\frac{1}{\sqrt{1+\frac{x^{2}}{3}}}
\end{gathered}
$$

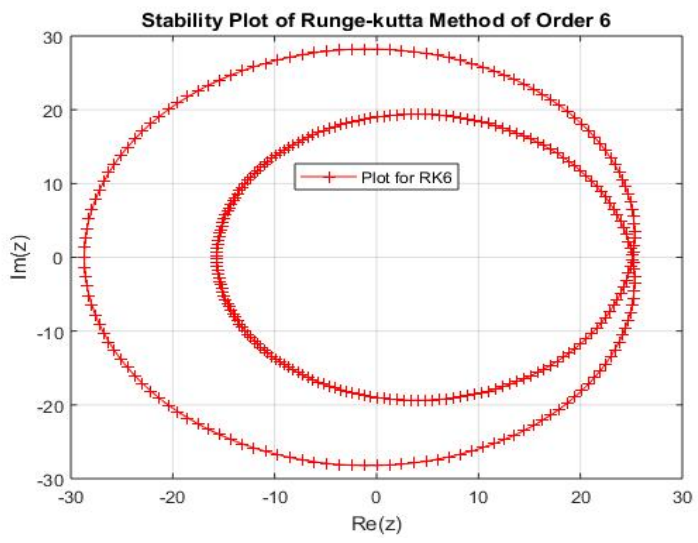

Figure 5. Stability Region for Runge-kutta Method of Order 6

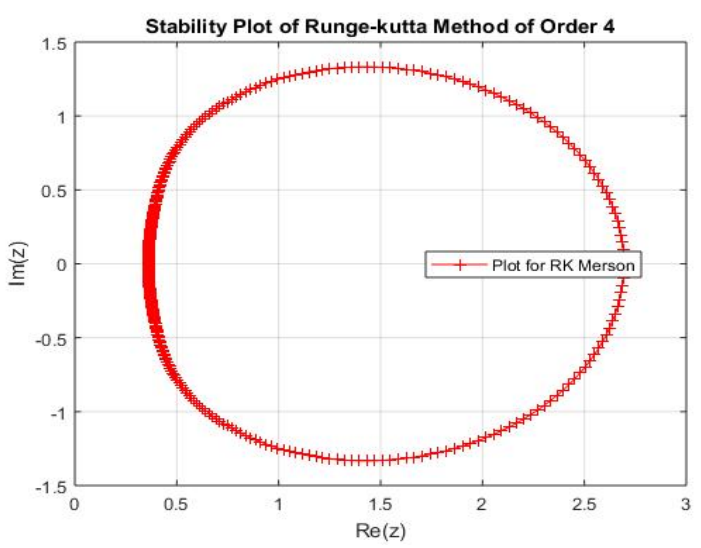

Figure 6. Stability Region for Merson's Method

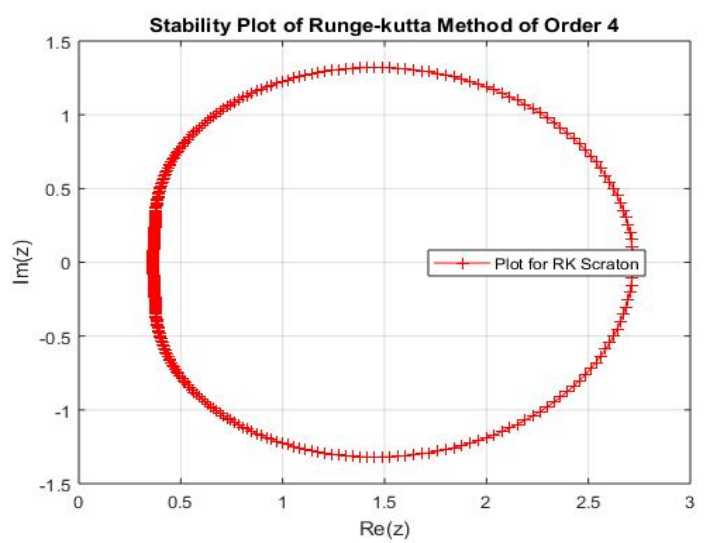

Figure 7. Stability Region for Scraton's Method

Example 4: Van der Pol Singular Problem [11]

$$
\begin{gathered}
y^{\prime \prime}=\frac{y^{\prime}\left(1-y^{\prime 2}\right)-y}{\mu} ; \\
y(0)=2, y^{\prime}(0)=-\frac{2}{3}+\frac{10}{81} \mu-\frac{292}{2187} \mu^{2} \\
-\frac{1814}{19683} \mu^{3} ; \mu=10^{-1} .
\end{gathered}
$$

The methods considered in this work were used to approximate the problem over the interval [0,0.55139] for $h=10^{-3}$. 
Table 4. Table of Absolute Errors for Example 3 Using different $h$

\begin{tabular}{|c|c|c|c|c|c|c|c|c|c|}
\hline \multirow[t]{2}{*}{$h$} & & \multicolumn{8}{|c|}{$y(x)$} \\
\hline & & RK2 & RK3 & RK4 & RK Nyström & RK Merson & RK Scraton & RK England & RK8 \\
\hline \multirow{3}{*}{$\frac{1}{7}$} & 0.2857 & $2.4565 \times 10^{-2}$ & $9.7182 \times 10^{+6}$ & $2.7524 \times 10^{-2}$ & $3.4496 \times 10^{-2}$ & $4.1169 \times 10^{-2}$ & $3.5739 \times 10^{-2}$ & $2.7525 \times 10^{-2}$ & $1.1722 \times 10^{-1}$ \\
\hline & 0.5714 & $2.8265 \times 10^{-2}$ & $9.7182 \times 10^{+6}$ & $3.1850 \times 10^{-2}$ & $3.8832 \times 10^{-2}$ & $4.54985 \times 10^{-2}$ & $3.9917 \times 10^{-1}$ & $3.1851 \times 10^{-2}$ & $1.2281 \times 10^{-1}$ \\
\hline & 0.8571 & $1.0144 \times 10^{-2}$ & $9.7182 \times 10^{+6}$ & $1.4411 \times 10^{-2}$ & $2.1093 \times 10^{-2}$ & $2.7780 \times 10^{-2}$ & $4.0945 \times 10^{-1}$ & $1.4112 \times 10^{-2}$ & $1.0723 \times 10^{-1}$ \\
\hline \multirow{3}{*}{$\frac{1}{14}$} & 0.2857 & $2.9836 \times 10^{-2}$ & $1.2148 \times 10^{+6}$ & $3.0700 \times 10^{-2}$ & $3.2486 \times 10^{-2}$ & $3.4150 \times 10^{-2}$ & $2.1129 \times 10^{-1}$ & $3.0701 \times 10^{-2}$ & $5.4061 \times 10^{-2}$ \\
\hline & 0.4286 & $3.4167 \times 10^{-2}$ & $1.2148 \times 10^{+6}$ & $3.5075 \times 10^{-2}$ & $3.0686 \times 10^{-2}$ & $3.8524 \times 10^{-2}$ & $2.3129 \times 10^{-1}$ & $3.5075 \times 10^{-2}$ & $5.9065 \times 10^{-2}$ \\
\hline & 0.6429 & $2.8969 \times 10^{-2}$ & $1.2148 \times 10^{+6}$ & $2.9944 \times 10^{-2}$ & $3.1729 \times 10^{-2}$ & $3.3393 \times 10^{-2}$ & $2.4973 \times 10^{-1}$ & $2.9944 \times 10^{-2}$ & $5.5316 \times 10^{-2}$ \\
\hline \multirow{3}{*}{$\frac{1}{21}$} & 0.4286 & $3.4837 \times 10^{-2}$ & $3.5993 \times 10^{+5}$ & $3.5246 \times 10^{-2}$ & $3.6042 \times 10^{-2}$ & $3.6782 \times 10^{-2}$ & $1.7714 \times 10^{-1}$ & $3.5246 \times 10^{-2}$ & $4.6596 \times 10^{-2}$ \\
\hline & 0.6667 & $2.8266 \times 10^{-2}$ & $3.5999 \times 10^{+5}$ & $2.8707 \times 10^{-2}$ & $2.9504 \times 10^{-2}$ & $3.0243 \times 10^{-2}$ & $1.9637 \times 10^{-1}$ & $2.8707 \times 10^{-2}$ & $4.1652 \times 10^{-2}$ \\
\hline & 0.9048 & $9.3712 \times 10^{-3}$ & $3.5993 \times 10^{+5}$ & $9.8446 \times 10^{-3}$ & $1.0641 \times 10^{-2}$ & $1.1138 \times 10^{-2}$ & $1.9780 \times 10^{-1}$ & $9.8446 \times 10^{-3}$ & $2.4823 \times 10^{-2}$ \\
\hline$\overline{\text { Cmpt Time (s) }}$ & & 0.0025 & 0.0021 & 0.0035 & 0.0059 & 0.0045 & 0.0077 & 0.0051 & 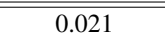 \\
\hline
\end{tabular}

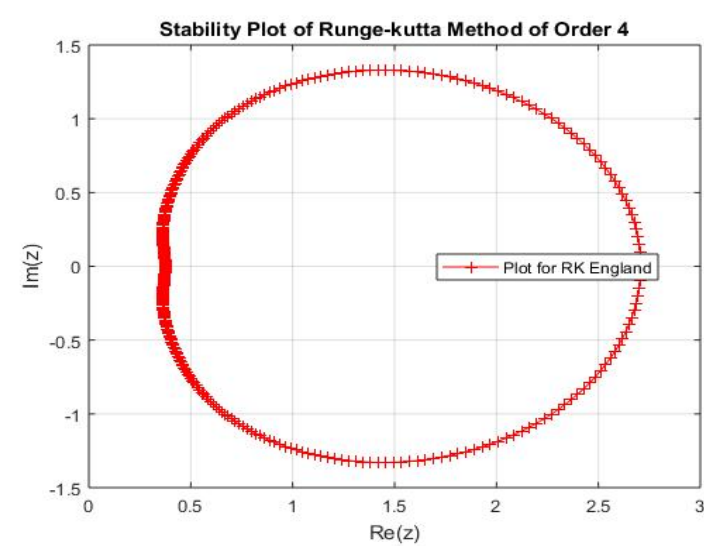

Figure 8. Stability Region for England's Method

Table 5. Numerical Results for Example 4 at $x=0.55139$

\begin{tabular}{c|c|c|c}
$h$ & Method & $y(x)$ & Cmpt. Time (s) \\
\hline \hline & RK2 & 0.625821430245524 & 0.0025 \\
& RK3 & 0.625821606669667 & 0.0019 \\
& RK4 & 0.625821602543592 & 0.0065 \\
$10^{-3}$ & RK Nyström & 0.625821602551168 & 0.018 \\
& RK Merson & 0.625821602551009 & 0.0028 \\
& RK Scarton & -0.031597285014076 & 0.025 \\
& RK England & 0.625821602543570 & 0.0021 \\
& RK 8 & 0.642280546551478 & 0.063 \\
\hline \hline
\end{tabular}

\section{Discussion of Results and Conclusion}

It is worthy to note that numerical methods were programmed via MATLAB 9.2 version on a personal computer with the following specifications:

- System name- HP Pavilion x360 Convertible

- Processor-Intel(R) Core(TM) i3-7100U CPU @ 2.40GHz

- Installed memory (RAM)- 8.00GB

- System Type- 64-bits Operating System, x64-based processor
- Operating system- Windows 10

Cmpt time is the computer computation time measured in seconds(s). This paper presents the performances of different orders of Runge-Kutta methods in the integration of singular Lane-Emden equations. From Tables 2-4, It could be observed that the Runge-Kutta method of order 4 performs the best in terms of accuracy. The methods of order 3, Scraton's, and order 8 were found to have failed in the solution of singular problems in ordinary differential equations. The England's method performs more satisfactorily as it shows some competitive strength against the order 4 method. It could be concluded that the Runge-Kutta method of order 4 outperforms all other methods under consideration and this property makes it the most stable and accurate of the Runge-Kutta methods.

\section{Acknowledgments}

The authors thank the referees and editor for their valuable comments and suggestions.

\section{References}

[1] A. Huta, "Contribution à la formule de sixieme ordre dans la methode de Runge-kutta-Nyström", Acta Fac Rerum Natu Univ. Comenian Math. 2 (1957) 21.

[2] R. Qu \& R. P. Agarwal, "A collocation method for solving a class of singular non-linear two-point boundary value problems", Journal Computer Application Mathematics 83 (1997) 147.

[3] O. Koch, K. Peter \& B. W. Ewa, "The Implicit Euler method for the numerical solution of singular initial value problems", Applied Numerical Mathematics 34 (2000) 231

[4] A. M. Wazwaz, "A new algorithm for solving differential equation of Lane-Emden type", Applied Mathematics and Computation 118 (2001) 287.

[5] A. M. Wazwaz, "Adomian decomposition method for a reliable treatment of the Emden-Fowler equation", Applied Mathematics and Computation 61 (2005) 543.

[6] H. Musa, S. Ibrahim \& M. Y. Waziri, "A Simplified Derivation and Analysis of Fourth Order Runge Kutta Method", International Journal of Computer Applications 9 (2010) 51.

[7] P. Roul, "A new efficient recursive technique for solving singular boundary value problems arising in various physical models", The European Physical Journal Plus 131 (2016), doi.org/10.1140/epjp/i2016-16105-8. 
[8] A. Abbas Al-Shimmary, "Solving Initial Value Problem Using Rungekutta $6^{\text {th }}$ Order Method", ARPN Journal of Engineering and Applied Sciences 12 (2017) 3953.

[9] P. Roul \& K. Thula, "A fourth order B-spline collocation method and its error analysis for Bratu-type and Lane-Emden problems", International Journal of Computer Mathematics 96 (2017) 85.

[10] H. Madduri, \& P. Roul, "A fast-converging iterative scheme for solving a system of Lane-Emden equations arising in catalytic diffusion reactions", Journal of Mathematical Chemistry 57 (2018) 570

[11] P. Roul, "Doubly singular boundary value problems with derivative dependent source function: A fast converging iterative approach", Journal of Math Methods Application Science 42 (2018) 354.

[12] M. O. Ogunniran, Y. Haruna, \& R. B. Adeniyi, "Efficient $k$-derivative Methods for Lane-Emden Equations and Related Stiff Problems", Nigerian Journal of Mathematics and Applications 28 (2019) 1.

[13] M. O. Ogunniran \& R. B. Adeniyi, "New Basic Rational Approximation Method for Solving Singular Initial Value Problems of Ordinary Differential Equations", ABACUS (Mathematics Science Series) 46 (2019) 119.

[14] M. O. Ogunniran, "A Class of Block Multi-Derivative Numerical Integrator for Singular Advection Equations", Journal of the Nigerian Society of
Physical Sciences 1 (2019) 62.

[15] M. O. Olayiwola, "Solutions of Emden-Fowler Type Equations by Variational Iteration Method", Cankaya University Journal of Science and Engineering 16 (2019) 1.

[16] M. O. Olayiwola \& A. Adegoke, "Homotopy Perturbation with Laplace Transforms Method for Solving Singular Initial Value Problems" Nigerian Journal of Mathematics and Applications 28 (2019) 65.

[17] P. Roul, K. Thula \& V. Goura, "An optimal sixth-order quartic B-spline collocation method for solving Bratu-type and Lane-Emden-type problems", Journal of Computational and Applied Mathematics 359 (2019) 182.

[18] P. Roul, H. Madduri \& R. Agarwal, "A fast-converging recursive approach for Lane-Emden type initial value problems arising in astrophysics", Journal of Math Methods Application Science 42 (2019) 2613.

[19] G. Singh, A. Garg, V. Kanwar \& H. Ramos, "An Efficient Optimized Adaptive Step-size Hybrid Block Method for Integrating Differential Systems", Applied Mathematics and Computation, 362 (2019) 124567.

[20] J. D. Lambert, Computational Methods in Ordinary Differential Equations, John Wiley and Sons, New York, 1991. 